\title{
Some Theorems on Tensor Composite Graphs*
}

\author{
Michael F. Capobianco**
}

(September 15, 1970)

\begin{abstract}
If a graph (digraph) is isomorphic to the tensor product of two graphs (digraphs) it is said to be a tensor composite graph (digraph). If not, it is said to be tensor prime. Several theorems giving various properties of tensor composite graphs and digraphs are presented. Among those dealing with (undirected) graphs is the result that any tree is tensor prime. This does not hold for digraphs. An example is given of a tensor composite digraph which is an unoriented tree. It is proved that a tensor composite digraph which is an oriented tree (an arborescence) does not exist. Some applications are presented.
\end{abstract}

Key words: Digraphs; graphs; products; tensor; trees.

\section{Introduction}

We refer to Ore [8], ${ }^{1}$ Berge [1], and Harary et al. [4], for the usual definitions of graph, digraph (directed graph) and related terms. The tensor product of two graphs $G_{u}$ and $G_{v}$ is denoted by $G_{u} \otimes G_{v}$ and defined as follows: If $V_{u}$ and $V_{v}$ are the sets of vertices of $G_{u}$ and $G_{v}$ respectively, then the set of vertices of $G_{u} \otimes G_{v}$ is $V_{u} \times V_{v}$. Two vertices $\left(u_{1}, v_{1}\right),\left(u_{2}, v_{2}\right)$ of $G_{u} \otimes G_{v}$ are adjacent if and only if $u_{1}$ and $u_{2}$ are adjacent in $G_{u}$, and $v_{1}$ and $v_{2}$ are adjacent in $G_{v}$. If $G_{u}$ and $G_{v}$ are digraphs then $\left(u_{1}, v_{1}\right)$ is adjacent to $\left(u_{2}, v_{2}\right)$ if and only if $u_{1}$ is adjacent to $u_{2}$ and $v_{1}$ is adjacent to $v_{2}$.

A number of papers, $[2,5,6,9]$, have studied various problems of characterizing the component graphs $G_{u}$ and $G_{v}$ so that $G_{u} \otimes G_{v}$ has certain properties. In this paper, as in an earlier one [3], we focus our attention on the structure of any graph which is isomorphic to the tensor product of two graphs, and investigate its properties. Such a graph (digraph) is called tensor composite. If there do not exist graphs $G_{u}$ and $G_{v}$ such that $G \cong G_{u} \otimes G_{v}$ then $G$ is said to be tensor prime.

\section{Main Theorems}

Our first theorem lists a number of necessary conditions for a graph to be tensor composite. We require the following definition of the distance between two lines of a graph. Let $l_{1}$ be the line joining $u$ and $v$ (written $u \sim v$ ), and let $l_{2}$ be $w \sim x$. The distance between $l_{1}$ and $l_{2}$, denoted by $d\left(l_{1}, l_{2}\right)$, is the length of the shortest path joining any vertex of $l_{1}$ with any vertex of $l_{2}$.

THeorem 1: Let $\mathrm{G}$ be a tensor composite graph with $\mathrm{n}$ vertices and l lines $(l>0)$ then $(1) \mathrm{n}=\mathrm{n}_{\mathrm{u}} \mathrm{n}_{\mathrm{v}}$ where $\mathrm{n}_{\mathrm{u}}$ and $\mathrm{n}_{\mathrm{v}}$ are integers greater than 1 such that for all vertices $\mathrm{v}$ of $\mathrm{G}, \mathrm{d}(\mathrm{v}) \leqslant\left(\mathrm{n}_{\mathrm{u}}-1\right)\left(\mathrm{n}_{\mathrm{v}}-1\right)$ where $\mathrm{d}(\mathrm{v})$ is the degree of $\mathrm{v},(2) l \leqslant 2\left(\begin{array}{c}\mathrm{n}_{\mathrm{u}} \\ 2\end{array}\right)\left(\begin{array}{c}\mathrm{n}_{\mathrm{v}} \\ 2\end{array}\right)(3) l=2 \mathrm{k}$, for some $\mathrm{k}=1,2, \ldots \ldots,\left(\begin{array}{c}\mathrm{n}_{\mathrm{u}} \\ 2\end{array}\right)\left(\begin{array}{c}\mathrm{n}_{\mathrm{v}} \\ 2\end{array}\right)$ and these l lines can be listed in $\mathrm{k}$ pairs such that for each such pair $l_{1}, l_{2}, \mathrm{~d}\left(l_{1}, l_{2}\right)>1$.

Proof: (1) Let $G$ be isomorphic to $G_{u} \otimes G_{v}$ and let $n_{u}$ and $n_{v}$ be the number of vertices of $G_{u}$ and $G_{v}$, respectively. Then $n_{u}>1, n_{v}>1$, for otherwise $l=0$, and furthermore it is clear that $n_{u} n_{v}=n$. For the vertices of $G_{u} \otimes G_{v}$ we use the abbreviation $\left(u_{i}, v_{j}\right)=u_{i} v_{j}$. Now since $u_{i}+u_{i}$ and $v_{i}+v_{i}$ for any $i$,

AMS subject classification. Primary 05C20, Secondary 05C05.

An invited paper.

Present address: St. John's University, Jamaica, New York 11432.

${ }^{1}$ Figures in brackets indicate the literature references at the end of this paper. 
$u_{i} v_{j}$ is adjacent with at most $\left(n_{u}-1\right)\left(n_{v}-1\right)$ vertices. Hence $d(v) \leqslant\left(n_{u}-1\right)\left(n_{v}-1\right)$ for all $v$ in $G$. (2) $l=\frac{1}{2} \sum_{v \in G} d(v) \leqslant \frac{1}{2} \sum_{v \in G}\left(n_{u}-1\right)\left(n_{v}-1\right) \leqslant \frac{1}{2} n_{u} n_{v}\left(n_{u}-1\right)\left(n_{v}-1\right)=2\left(\begin{array}{c}n_{u} \\ 2\end{array}\right)\left(\begin{array}{c}n_{v} \\ 2\end{array}\right)$. (3) Suppose $u_{1} v_{1} \sim u_{2} v_{2}$. Then $u_{1} v_{2} \sim u_{2} v_{1}$. Also $u_{1} v_{1} \nmid u_{1} v_{2}, u_{1} v_{1} \nmid u_{2} v_{1}, u_{2} v_{2} \nmid u_{1} v_{2}, u_{2} v_{2} \nmid u_{2} v_{1}$. Hence the distance between these two lines is greater than 1 .

The above theorem has a parallel for digraphs with a similar proof [3].

Note that if $l$ is the number of lines in a tensor composite graph and $l_{u}$ and $l_{v}$ are the numbers of lines in the two components graphs then $l=2 l_{u} l_{v}$. We use this fact in an example which shows that the conditions in theorem 1 are not sufficient, Let $G$ be the graph shown below.

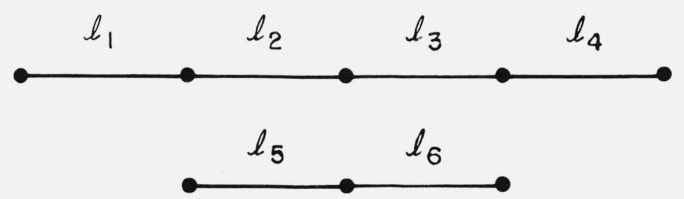

Then $n=8=4 \times 2, d(v) \leqslant 2 \leqslant 3, l=6, d\left(l_{1}, l_{4}\right)>1, d\left(l_{2}, l_{6}\right)>1, d\left(l_{3}, l_{5}\right)>1$. However, for $G$ to be tensor composite $G_{u}$, say, would have to have 4 vertices and 3 lines, and $G_{v}, 2$ vertices and 1 line. The only possibilities are shown below, and $G$ is none of these.

Gu:

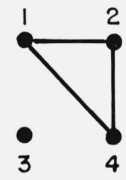

Gu $\otimes$ Gv:
Gv:

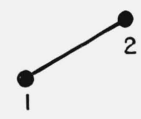

Gv:

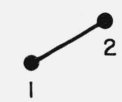

Gu:

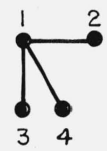

Gu $\otimes G v:$
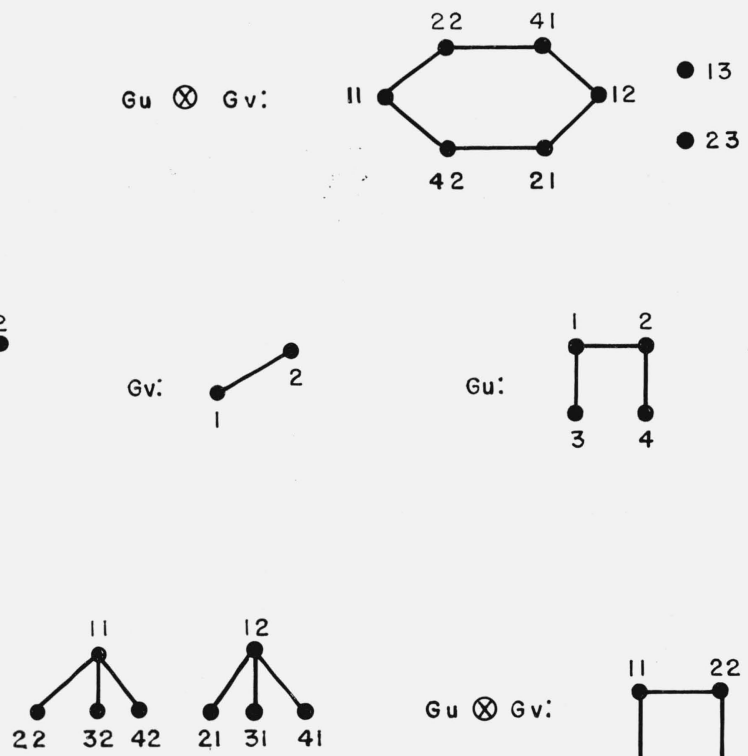

Gu:

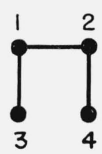

Gv:

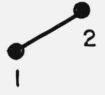

Gu $\otimes G v:$
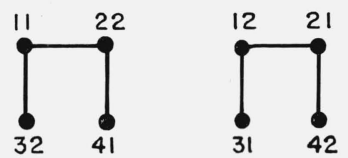

The problem of characterizing tensor composite graphs and digraphs seems quite complex and remains unsolved. A characterization of digraphs having a prime number of lines was given in [3]. The remainder of this paper will be devoted to two nonexistence theorems and their applications.

THEOREM 2: No tree is tensor composite.

Proof: We use two lemmas.

Lemma 1: If $\mathrm{G}_{\mathrm{u}}$ or $\mathrm{G}_{\mathrm{v}}$ has a cycle, then so does $\mathrm{G}_{\mathrm{u}} \otimes \mathrm{G}_{\mathrm{v}}$. 
Proof: Let

Then if $k$ is even,

$$
u_{1} \sim u_{2} \sim u_{3} \sim \ldots ., \sim u_{k} \sim u_{1}, v_{1} \sim v_{2}
$$

$$
u_{1} v_{1} \sim u_{2} v_{2} \sim u_{3} v_{1} \sim u_{4} v_{2} \sim v_{5} v_{1} \sim \ldots \sim u_{k} v_{2} \sim u_{1} v_{1}
$$

so that $G_{u} \otimes G_{v}$ has a cycle. If $k$ is odd then

$$
u_{1} v_{1} \sim u_{2} v_{2} \sim u_{3} v_{1} \sim u_{4} v_{2} \sim \ldots \sim u_{k} v_{1} \sim u_{1} v_{2} \sim u_{2} v_{1} \sim \ldots \sim u_{k} v_{2} \sim u_{1} v_{1}
$$

so that $G_{u} \otimes G_{v}$ again has a cycle.

LEMMa 2: The tensor product of two forests is not connected.

Proof: This lemma follows, in part, from Weichel's theorem 1, [9], which states that a tensor composite graph is connected if and only if the component graphs are connected and at least one of them has an odd cycle. For the sake of completeness, we give an independent proof of the lemma here.

Consider $u_{1} v_{1}$ and $u_{1} v_{2}$ with $v_{1} \sim v_{2}$. We claim that there is no path between $u_{1} v_{1}$ and $u_{1} v_{2}$. There can not be a path between $u_{1}$ and $u_{1}$ since this is a cycle and its existence would contradict the fact that $G_{u}$ is a forest. Therefore, the only way one can have a path between $u_{1} v_{1}$ and $u_{1} v_{2}$ is if this path has the form

$$
u_{1} v_{1} \sim u_{2} v_{j} \sim u_{1} v_{k} \sim u_{2} v_{m} \sim \ldots \sim u_{1} v_{2}
$$

We examine the possibilities. Of course $j \neq 1, k \neq 1$. If $j=2$ then $k \neq 2$. Let $k=3$. Then $m \neq 1$ for otherwise there would exist a cycle containing $v_{1}$. It is also clear that $m \neq 2$. Therefore, $m=4$. But this implies the existence of a cycle containing $v_{2}$. Hence we must have $j=3$. However, this implies the existence of a path between $v_{1}$ and $v_{2}$ other than $v_{1} \sim v_{2}$, which would mean that $v_{1}$ and $v_{2}$ are contained in a cycle. This final contradiction proves lemma 2.

Since a tree is a connected graph without cycles, the theorem follows immediately.

Lemma 2 has a parallel for digraphs. One talks about semicycles instead of cycles, and weakly connected instead of connected. Thus if we understand that a forest is a digraph without semicycles than we can prove the following result.

LEMMA 2': The tensor product of two forests is not weakly connected.

Lemma 1, however does not hold for digraphs, as can be seen by the following example.
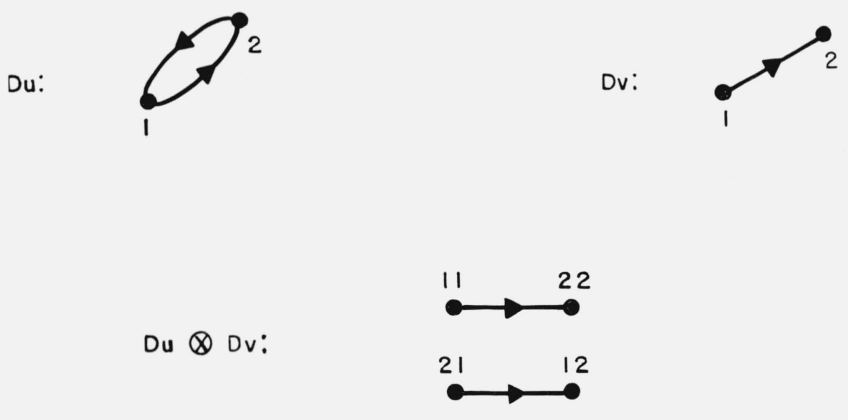

Hence the proof of theorem 2 does not go through for digraphs. In fact, the theorem is not true, as can be seen by the example below. 
Note that in this example the tree obtained is an unoriented one. One immediately asks if an oriented tree (an arborescence) can be tensor composite. The answer is given in the next theorem. THEOREM 3: Except for the trivial graph (a single point), any arborescence is tensor prime.

Proof: Consider a nontrivial tensor composite digraph $D$ in which $u_{1} v_{1}$ has indegree 0 . Then $u_{i} v_{j}+u_{1} v_{1}$ for all $i$ and $j$. Therefore, either $u_{i} \nmid u_{1}$ for all $i$ or $v_{j} \nmid v_{1}$ for all $j$. Hence for some $k \neq 1$ either $u_{1} v_{k}$ or $u_{k} v_{1}$ has indegree 0 . Since an arborescence requires exactly one point with indegree 0 , $D$ can not be an arborescence.

Using a dual argument it is easy to see that no nontrivial tensor composite digraph can have exactly one point with outdegree 0 .

Du:

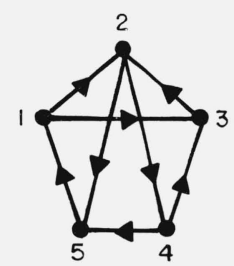

Dv:
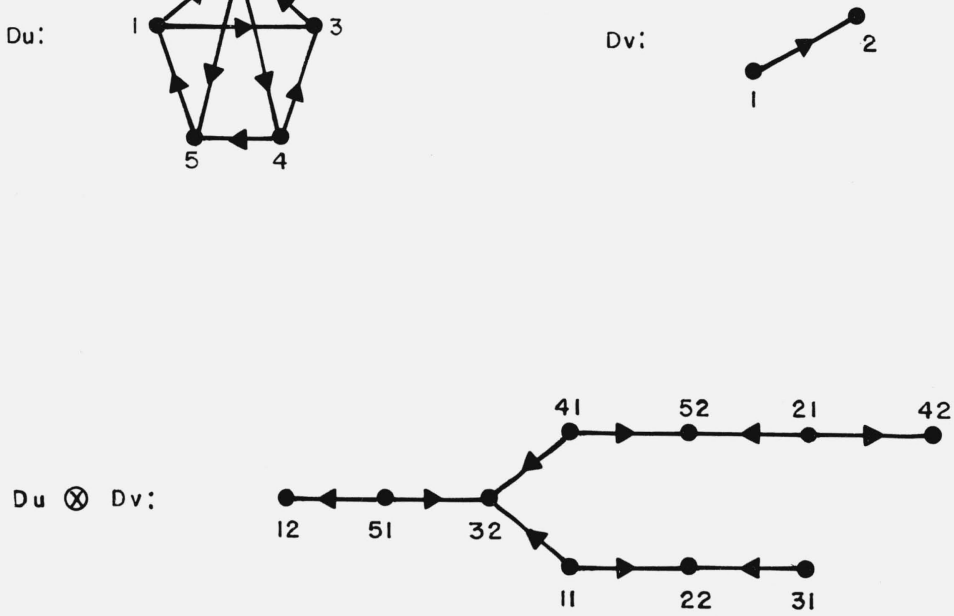

\section{Some Applications}

We present applications of theorems 2 and 3. Theorem 2 is used in the form: Any connected tensor composite graph has at least one cycle.

Consider a message which for security purposes is to be transmitted in two parts, possibly a text and a key. We have a group of $n$ agents among whom part 1 can be transmitted, and a group of $m$ agents among whom part 2 can be transmitted. We represent these two groups by two graphs in which the vertices are the agents and two vertices are adjacent if and only if the two agents they represent communicate with each other. The tensor product of these two graphs then represents the communication pattern for the entire message among all possible "teams" of two agents each. Theorem 2 tells us that if things are arranged in such a way that the message can get from any agent team to any other (the tensor product is connected) then there must exist at least three teams which can experience feedback in the sense that a message can be returned to them (the tensor product has at least one cycle).

Theorem 3 can be applied to a situation in which two sets of players compete in tournaments. We represent each set by a digraph in which the vertices are the players and $u$ is adjacent to $v$ if and only if $u$ defeats $v$. In the case of a tie we let $u$ and $v$ be adjacent to each other. Now form all possible teams, or coalitions, of two players, one from the first set and one from the second set. Assuming that the strengths of the players are additive, the tensor product will represent the "victory-defeat" pattern for these coalitions. From the proof of theorem 3 we see that there can 
not be exactly one undefeated coalition. Either there is none, or there are more than one. That the latter is possible can be seen by our last two examples. The former is illustrated below.

Du:

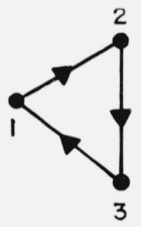

Dv:

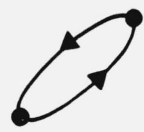

$D u \otimes D v:$

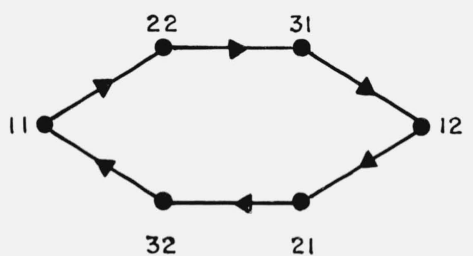

\section{References}

[1] Berge, C., Theory of Graphs and its Applications (Wiley 1962).

[2] Capobianco, M. F., Tournaments and tensor products of digraphs, SIAM J. App. Math. 15, 624-626 (1967).

[3] Capobianco, M. F., Tensor products of digraphs and the structure of groups of pairs, Bull. Math. Biophysics 31 , 319-326 (1969).

[4] Harary, F., Norman, R., Cartwright, D., Structural Models (Wiley 1965).

[5] Harary, F., and Trauth, C., Connectedness of products of two directed graphs, SIAM J. App. Math. 14, 250-254 (1966).

[6] McAndrew, M., On the product of directed graphs. Proc. Amer. Math. Soc. 14, 600-606 (1963).

[7] Miller, D., The categorical product of graphs, Can. J. Math. 20, 1511-1521 (1968).

[8] Ore, O., Theory of graphs, Amer. Math. Soc. (1962).

[9] Weichsel, P., The kronecker product of graphs, Proc. Amer. Math. Soc. 13, 47-52 (1962).

(Paper 74B4-333) 